\title{
A Low-Cost Analytical Method for the Morris Water Maze Task using Image $\mathbf{J}^{\dagger}$
}

\author{
Apsorn SATTAYAKHOM ${ }^{1,3}$ and Phanit KOOMHIN K,, $^{2, *}$ \\ ${ }^{1}$ School of Allied Health Sciences, Walailak University, Nakhon Si Thammarat 80160, Thailand \\ ${ }^{2}$ School of Medicine, Walailak University, Nakhon Si Thammarat 80160, Thailand \\ ${ }^{3}$ Center of Excellence in Innovation on Essential Oil, Walailak University, \\ Nakhon Si Thammarat 80160, Thailand
}

('Corresponding author's e-mail: phanit.ko@mail.wu.ac.th)

Received: 5 April 2019, Revised: 13 July 2019, Accepted: 19 August 2019

\begin{abstract}
The Morris water maze, which has been used for more than 30 years, is one of the most famous learning and memory tasks among animals. This method is robust and reliable, and it can be carried out anywhere with little effort. Nowadays, there are many companies creating analysis software to easily analyze the Morris water maze results. However, these softwares are costly and may be difficult for researchers in developing countries especially with limited research funds. Only escape latency and time spent in the target quadrant can be manually obtained after video review. To resolve this problem and to extend the ability to detect learning and memory impairment in the Morris water maze, we provide a novel low-cost analysis method using common office tools combined with ImageJ software to analyze learning and memory impairment in rat or mouse models. In this study, we used the 2-vessel occlusion $(2 \mathrm{VO})$ rats and sham-operated control rats as learning and memory deficit rats and normal rats, respectively. Taken together, travel distance, swimming speed, and swimming traces can be acquired from this method, which will help researchers to investigate further impairment of learning and memory in animal models.
\end{abstract}

Keywords: Morris water maze task, ImageJ, Low-cost analysis method, Learning and memory impairment

\section{Introduction}

Learning and memory is one of the most interesting topics in the field of neuroscience. It helps us survive through difficult situations along with pre-trained data and adapt to fit each environment. Learning and memory may decline if the nervous system is not maintained in physiological working range $[1,2]$. To study the learning and memory mechanism, animals were used as models to explore the mechanisms and impairment processes or to find new drugs or alternative methods to improve impairment. The Morris water maze task is a famous task using rats or mice as animal models. Morris showed a practical way and reliable method to study learning and memory in rat models. The protocol has been used for more than 3 decades [3,4]. Nowadays, there are several companies offering analysis software which provides easy ways to get many important parameters to analyze behavioral impairment among animal models. However, if there is a limitation of budget as in developing countries, it is a pity that researchers can only get the results of a few parameters, such as escape latency and time spent in the target quadrant. To increase the chance in analyzing the behavioral impairments among animals, we

\footnotetext{
${ }^{\dagger}$ Presented at the International Conferences on Biomedical Sciences and Medical Technology 2019: June 20 - 21, 2019
} 
http://wjst.wu.ac.th

herein provide an alternative way to analyze the Morris water maze task with common office tools and ImageJ.

ImageJ is a NIH-supported public domain JAVA image processing program which is available for Windows, Mac OS, and Linux. It has plenty of functions to analyze images [5]. For the Morris water maze analysis, we can use scale function to calculate the pixels to a known unit, $\mathrm{cm}$ or $\mathrm{m}$. This low-cost method will extract important parameters from the Morris water maze task without the need of an expensive analysis software. The obtained parameters will increase sensitivity in detecting deficits not only in swimming performance but also in swimming strategy and motor weakness. This method needs only tools which can be easily found in any office. Travel distance, swimming speed, and swimming traces can be acquired from this method, which will benefit behavioral analysis especially with limited budget support.

\section{Materials and methods}

\section{Set-up}

With the conventional Morris water maze task, a ceiling-mounted digital camera and a computer are needed to record animal behavior during testing. The Morris water maze in this study was modified from a previous study in which the maze was a $2 \mathrm{~m}$ size circular swimming pool [6]. Various extra maze cues were placed at each quadrant of the maze. Twenty-five $\mathrm{cm}$ of water was filled in the maze with a submerged glass platform about $2 \mathrm{~cm}$ underneath the water surface. A ceiling-mounted video camera controlled by a desktop computer was used to record behavioral tasks. Learning and memory were evaluated from training sessions and probe tests. In the hidden-platform session and learning trial, each rat was given $120 \mathrm{~s}$ to swim in the maze to find the hidden target platform. Escape latency, which is the time spent by each rat to find the platform, was measured for four trials per day for 7 consecutive days. The location of the hidden platform was fixed at the same place in every trial. The probe test was performed on the $8^{\text {th }}$ day. During the probe test session, the platform was removed from the pool, and the rats were allowed to swim for the same duration as in the training trial. The time spent in the target quadrant where the platform was previously located in the hidden-platform session was recorded. A document scanner, a sketch pad, transparent sheets, and permanent markers are additional tools required. ImageJ software can be downloaded via https://imagej.nih.gov/ij (Figure 1).

(a)

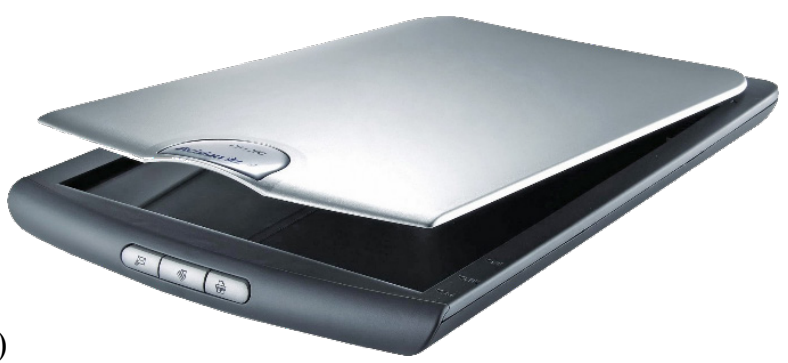

(b)

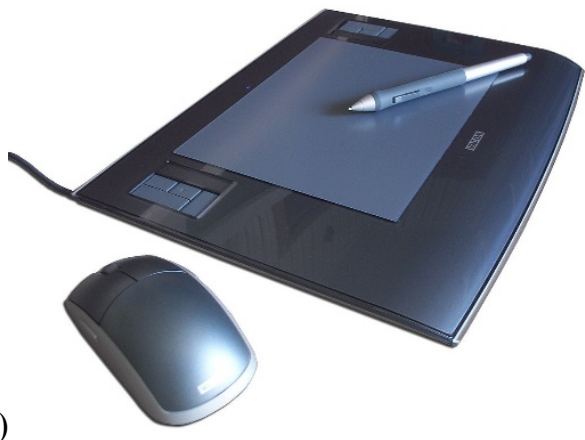

Figure 1 Office tools needed for behavioral analysis with ImageJ, a scanner (a) and a sketchpad (b). 
http://wjst.wu.ac.th

\section{Animals}

Sixteen male Spraque Dawley rats aged 8 weeks old were used as an animal model in this experiment. All animals were obtained from the National Laboratory Animal Centre, Mahidol University, Salaya, Nakornpathom, Thailand. The animals were housed in clean conventional cages with natural light/dark cycle with a constant room temperature of $25{ }^{\circ} \mathrm{C}$. Food and water were given ad libitum. Experimental procedures were approved by Siriraj Animal Care and Use Committee, Faculty of Medicine Siriraj Hospital, Mahidol University, Thailand. Two-vessel occlusion was conducted as previously described. For the procedure, a cervical incision was performed, and the common carotid arteries were revealed. The vagus nerves were split from the common carotid arteries, and the vessels were tied using suture thread. Sham-operated control rats and 2-vessel occlusion (2VO) rats were used to demonstrate further behavioral analyses. The sham-operated control rats represented normal rats, which showed normal learning and memory processes. The $2 \mathrm{VO}$ rats represented learning and memory deficit rats [7].

(a)

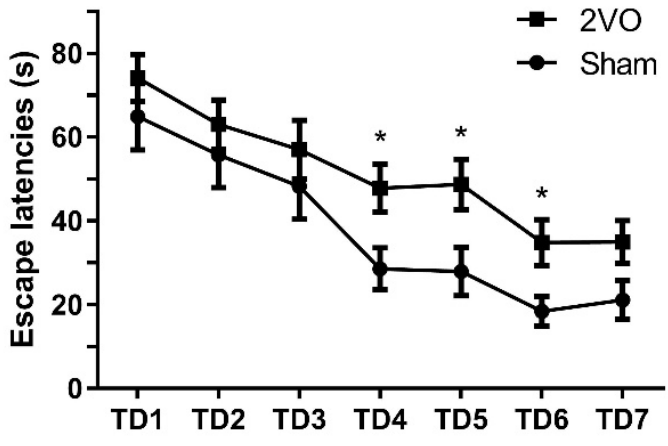

(b)

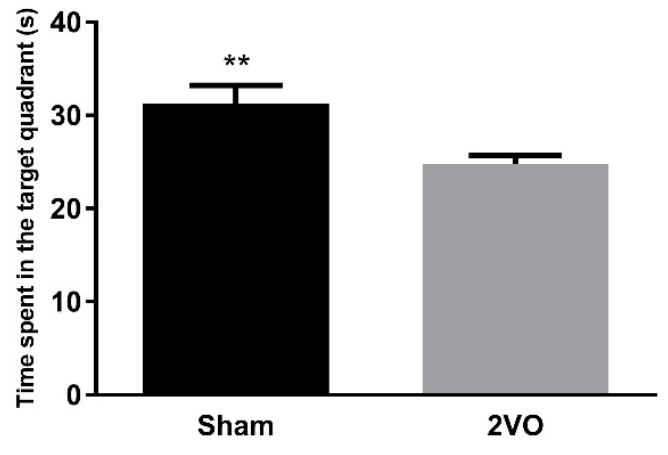

Figure 2 Conventional parameters obtained from the Morris water maze task. Escape latencies measured spent time to the hidden target platform underneath the water surface (a). Probe test measured time spent in the target quadrant (b). Sham $=$ sham-operated control rats, $2 \mathrm{VO}=2$-vessel occlusion rats, $\mathrm{TD}=$ training day. ${ }^{*} \mathrm{p}<0.05,{ }^{*} \mathrm{p}<0.01$ compared with the other group, $\mathrm{n}=8$.

(a)

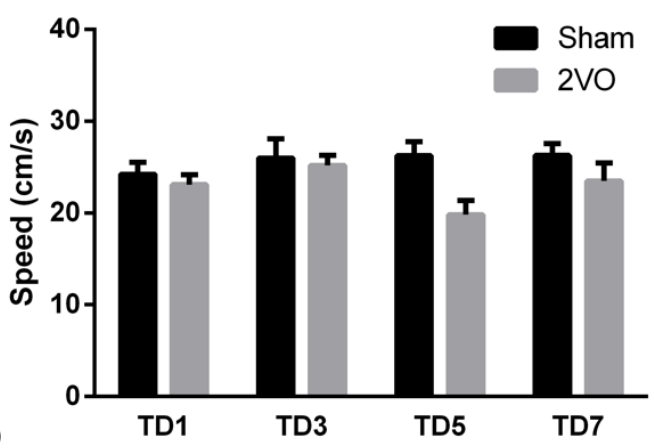

(b)

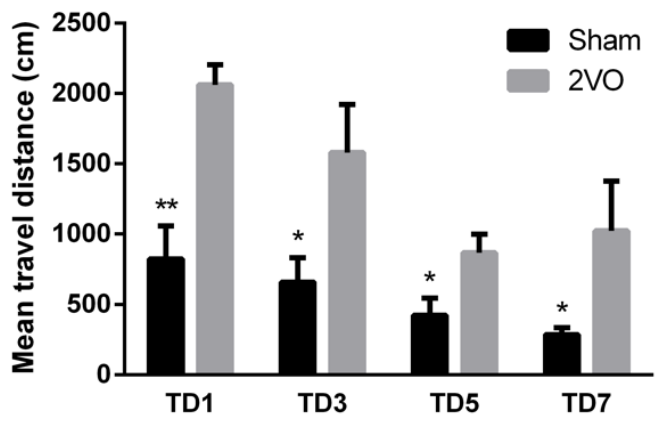

Figure 3 Additional parameters obtained from ImageJ. Average speeds calculated from travel distances divided by escape latencies (a). Travel distances calculated from pixel counts calibrated to known distances (b). Sham $=$ sham-operated control rats, $2 \mathrm{VO}=2$-vessel occlusion rats, $\mathrm{TD}=$ training day. ${ }^{*} \mathrm{p}<$ $0.05, * * \mathrm{p}<0.01$ compared with the other group, $\mathrm{n}=8$. 
http://wjst.wu.ac.th

\section{Analysis}

Recorded task videos were played using video player software. The 2 parameters that can be manually recorded during video playing included escape latency and time spent in the target quadrant. During video playing, the swimming pattern was copied from a computer screen to transparent sheets. The border of the pool was marked as a known distance $(200 \mathrm{~cm})$ in the trace. The transparent sheets were then scanned and saved as photo extensions. The scanned traces were opened via ImageJ software, and a straight line was drawn from the marker border of each side to the other side. Next, the "Analyze" function was performed and "Set Scale" was chosen. During the straight line drawing, pixel distance, which reflects known distance of the water pool, was obtained. The known distance and length unit were filled to set scale for the software to analyze a real distance. A free-hand line was used to copy the swimming trace. The "Analyze" function and "Measure" tab were performed in order to send the results to a result window. Calibrated length of the swimming trace was shown in the "Result" window. The length suggested travel distance. Average speed can then be calculated using known distance and escape latency. To create swimming traces, a new image with a white background was generated. The "Image" and "Overlay" functions were performed and the trace photo was selected as an overlay. Using a freehand line, the swimming pattern was drawn, and then the overlay was removed after the drawing was finished. Traces were then saved as photo files and ready to be used as a trace result. All results were expressed as mean \pm SEM. The differences were analyzed using an unpaired student's t-test to determine the differences between the groups. Statistical significance was considered at $p<0.05$.

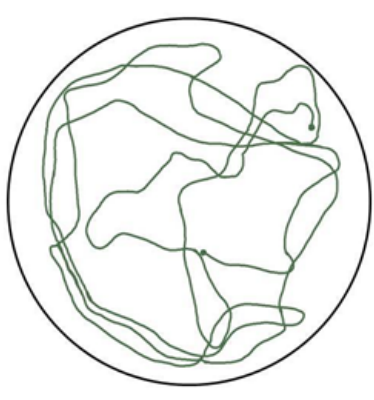

Sham rat $1^{\text {st }}$ day

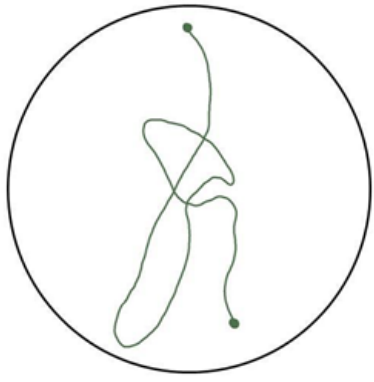

Sham rat $7^{\text {th }}$ day

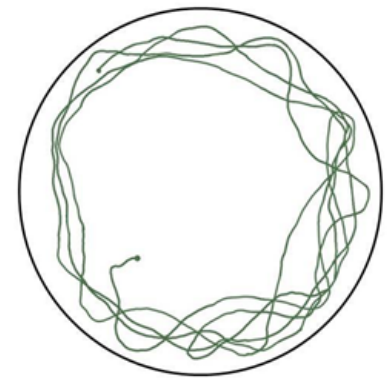

$2 \mathrm{VO}$ rat $1^{\text {st }}$ day

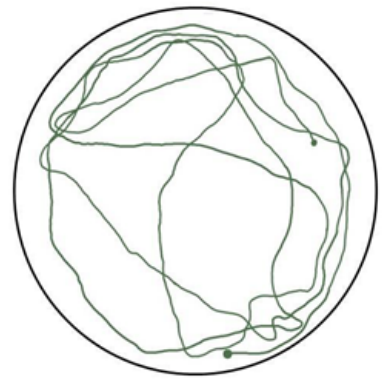

$2 \mathrm{VO}$ rat $7^{\text {th }}$ day

Figure 4 Representative swimming traces drawn from the scanned pattern. Sham-operated control rat and $2 \mathrm{VO}$ rat traces were drawn from the scanned pattern showing swimming path on $1^{\text {st }}$ day and $7^{\text {th }}$ day of training. Sham $=$ sham-operated control rats, $2 \mathrm{VO}=2$-vessel occlusion rats. 
http://wjst.wu.ac.th

\section{Results and discussion}

Escape latency and time spent in the target quadrant were manually measured during video viewing as shown in Figure 2. Escape latency showed learning and memory competency between the groups, and time spent in the target quadrant reflected retention memory after consecutive training. Learning and memory impairment can be observed from the $4^{\text {th }}$ day to the $7^{\text {th }}$ day of training in this $2 \mathrm{VO}$ model compared with the sham-operated control rats, and memory retention was also statistically impaired during the probe test for the $2 \mathrm{VO}$ rats. Speed and travel distance can be processed using ImageJ software. There is no difference in speed between the groups, suggesting no motor weakness in the $2 \mathrm{VO}$ rats when compared with the sham-operated control rats. Interestingly, the travel distance obtained from the analysis showed a difference between the groups. The $2 \mathrm{VO}$ rats showed a longer distance when compared with the sham-operated control rats, even when both shared the same swimming speed. Travel distance revealed that the 2VO rats swam without strategy, using a longer time and a longer distance to find the hidden platform (Figure 3). To confirm strategic swimming of the rats, swimming traces were the best way to reveal the swimming strategy. Swimming traces revealed that there was a vast difference in the shamoperated rats between the $1^{\text {st }}$ day and the $7^{\text {th }}$ day. In the last day of training, the rats spent less time to find the platform and directly swam to the hidden platform position. However, the 2VO rats swam randomly, suggesting deficiency of learning and memory which occurred in the training process as well (Figure 4). Swimming patterns showed the method that rats used to find the hidden platform. Nonspatial concentric pattern was used by the $2 \mathrm{VO}$ rats, indicating a lack of goal-directed swimming strategy [8]. Swimming traces during the probe test can be drawn to show the pattern of memory retention if needed.

\section{Conclusions}

Ordinary office tools combined with ImageJ software can be used to analyze the Morris water maze task or even other behavioral testing. Distance, speed, and travel traces are the important parameters decoded from the protocol. Thus, it is practical with limited budget.

\section{Acknowledgements}

We would like to thank Ms. Supaya Chandharakool for her graphic drawing and the Center of Excellence in Innovation on Essential Oil for the partial support given during the manuscript preparation. Finally, we would like to express our gratitude to Dr. Sompol Tapechum who owns the behavioral data used for the study.

\section{References}

[1] LD Hoz, SJ Martini and RGM Morris. Forgetting, reminding, and remembering: The retrieval of lost spatial memory. PloS Biol. 2004; 2, 1233-42.

[2] P Koomhin, A Sattayakhom, S Palipoch, C Punsawad and S Tapechum. Effects of non-spatial pretraining on learning and memory impairment detection in the Morris water maze. Walailak J. Sci. \& Tech. 2018; 15, 169-77.

[3] R D'hooge and PP De Deyn. Applications of the Morris water maze in the study of learning and memory. Brain Res. Rev. 2001; 36, 60-90.

[4] R Morris. Developments of a water-maze procedure for studying spatial learning in the rat. $J$. Neurosci. Meth. 1984; 11, 47-60.

[5] CA Schneider, WS Rasband and KW Eliceiri. NIH Image to ImageJ: 25 years of image analysis. Nat. Meth. 2012; 9, 671-5.

[6] P Koomhin, K Tilokskulchai and S Tapechum. Ceftriaxone improves spatial learning and memory in chronic cerebral hypoperfused rats. Sci. Asia 2012; 38, 356-63.

[7] E Farkas, PGM Luiten and F Bari. Permanent, bilateral common carotid artery occlusion in the rat: A model for chronic cerebral hypoperfusion-related neurodegenerative diseases. Brain Res. Rev. 2007; 54, 162-80. 
http://wjst.wu.ac.th

[8] S Dalm, J Grootendorst, ERD Kloet and MS Oitzl. Quantification of swim patterns in the Morris water maze. Behav. Res. Meth. Instrum. Comput. 2000; 32, 134-9. 